\title{
Laser Cooling below the One-Photon Recoil Energy by Velocity-Selective Coherent Population Trapping
}

\author{
A. Aspect, E. Arimondo, ${ }^{(a)}$ R. Kaiser, N. Vansteenkiste, and C. Cohen-Tannoudji \\ Laboratoire de Spectroscopie Hertzienne de l'Ecole Normale Supérieure et Collège de France, \\ F-75231 Paris Cedex 05, France \\ (Received 11 July 1988)
}

\begin{abstract}
We present a new laser-cooling scheme based on velocity-selective optical pumping of atoms into a nonabsorbing coherent superposition of states. This method has allowed us to achieve transverse cooling of metastable ${ }^{4} \mathrm{He}$ atoms to a temperature of $2 \mu \mathrm{K}$, lower than both the usual Doppler cooling limit ( 23 $\mu \mathrm{K})$ and the one-photon recoil energy $(4 \mu \mathrm{K})$. The corresponding de Broglie wavelength $(1.4 \mu \mathrm{m})$ is larger than the atomic-transition optical wavelength.
\end{abstract}

PACS numbers: $32.80 . P \mathrm{j}, 42.50 . \mathrm{Vk}$

The lowest temperature $T$ which can be achieved by the usual laser-Doppler-cooling method is given, for a two-level atom, by $k_{\mathrm{B}} T / 2=\hbar \Gamma / 4$, where $\Gamma$ is the spontaneous-emission rate from the excited atomic state (for $\mathrm{Na}, T \simeq 240 \mu \mathrm{K}) .{ }^{1}$ In order to reach lower temperatures, proposals based on Raman two-photon processes in a three-level atom have been presented, ${ }^{2,3}$ but the efficiency of Raman cooling has not yet been demonstrated. Recently, surprisingly low temperatures (around $40 \mu \mathrm{K}$ ) have been measured for sodium ${ }^{4}$ and tentatively interpreted in terms of a new friction mechanism. ${ }^{5}$ The recoil energy $(\hbar k)^{2} / 2 M$ for an atom with mass $M$ emitting a photon with momentum $\hbar \mathbf{k}$ represents another landmark in the energy scale for laser cooling. It has been suggested that optical pumping in translation space might be used to cool the translational degrees of freedom below this so-called recoil limit, by velocity-selective recycling in a trap. ${ }^{6}$ In this Letter, we present a mechanism of laser cooling below the onephoton recoil energy, based on optical pumping of both internal and translational atomic degrees of freedom. This velocity-selective process is based on coherent trapping of atomic populations ${ }^{7}$ and has allowed us to achieve a one-dimensional cooling of ${ }^{4} \mathrm{He}$ atoms in the triplet metastable state down to a temperature of about 2 $\mu \mathrm{K}$. This temperature is lower than both the Doppler cooling limit ( $23 \mu \mathrm{K}$ for 1D cooling) and the one-photon recoil energy $(4 \mu \mathrm{K})$.

Our scheme involves a closed three-level $\Lambda$ configuration where two degenerate ground Zeeman sublevels $g_{ \pm}$ $(m= \pm 1)$ are coupled to an excited level $e_{0}(m=0)$ by two counterpropagating $\sigma+$ and $\sigma$ - laser beams with the same frequency $\omega_{L}$ and the same intensity (solid lines of Fig. 1). For an atom at rest, two-photon Raman processes give rise to a nonabsorbing coherent superposition of $g_{+}$and $g_{-}$. If the atom is moving along $0 z$, the Raman resonance condition is no longer fulfilled as a consequence of opposite Doppler shifts on the two counterpropagating laser beams. This simple argument explains how the phenomenon of coherent population trapping can be velocity selective for appropriate laser configurations. ${ }^{8}$ Our cooling scheme consists of accumulating atoms in the zero-velocity nonabsorbing state where they remain trapped. To populate this state, we take advantage of momentum redistribution due to spontaneous emission, which allows certain atoms to be optically pumped from the absorbing velocity classes to the nonabsorbing state. Since the recoil of the last spontaneous-emission photon is part of the cooling mechanism, the one-photon recoil energy is not a limit and the final temperature is limited only by the coherent interaction time. Note also that, contrary to other cooling schemes, our mechanism, based on a Raman resonance condition, does not depend on the sign of the laser detuning.

A more rigorous analysis requires the introduction of both internal and translational quantum numbers. For example, the state $\left|e_{0}, p\right\rangle$ represents an atom in level $e_{0}$ with the value $p$ of $P_{z}^{\text {at }}$ ( $P^{\text {at }}$ is the atomic momentum). If we ignore spontaneous emission, $\left|e_{0}, p\right\rangle$ is coupled only to $\left|g_{-, p-\hbar k}\right\rangle$ (or $\left|g_{+}, p+\hbar k\right\rangle$ ) by stimulated emission of a $\sigma_{+}\left(\sigma_{-}\right)$laser photon carrying a momentum

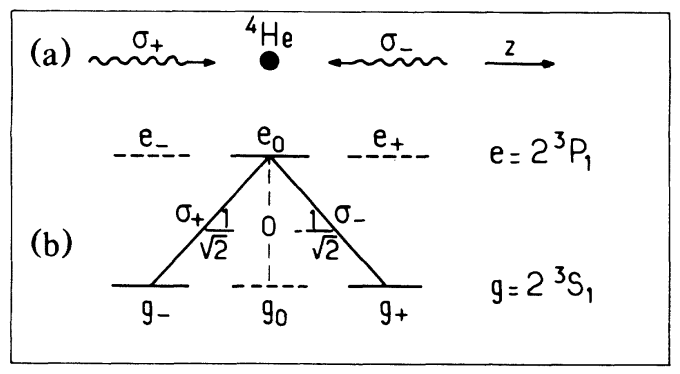

FIG. 1. (a) Two counterpropagating $\sigma_{+}$and $\sigma_{-}$polarized laser beams interact with ${ }^{4} \mathrm{He}$ atoms on the $2{ }^{3} S_{1}-2{ }^{3} P_{1}$ transition. (b) The Zeeman sublevels, and some useful ClebschGordan coefficients. Since the $e_{0} \leftrightarrow g_{0}$ transition is forbidden, all atoms are pumped into $g+$ and $g-$ after a few fluorescence cycles. These two levels are coupled only to $e_{0}$, and a closed three-level $\Lambda$ configuration is realized (solid lines). 
$+\hbar k(-\hbar k)$. We are thus led to introduce, for each value of $p$, a family $F(p)$ of three states $\left\{\left|e_{0}, p\right\rangle\right.$, $\left|g_{+}, p+\hbar k\right\rangle$, and $\left.\left|g_{-, p}-\hbar k\right\rangle\right\}$ which are coupled by the interaction Hamiltonian $V$ (Ref. 9):

$$
\left\langle g_{ \pm}, p \pm \hbar k|V| e_{0}, p\right\rangle=\mp\left(\hbar \omega_{1} / 2\right) \exp \left(i \omega_{L} t\right),
$$

where $\omega_{1}$ is the Rabi frequency associated with each laser and where the $\mp$ signs come from the ClebschGordan coefficients $e_{0} \leftrightarrow g_{+}$and $e_{0} \leftrightarrow g_{-}$(Fig. 1). Note that for $p \neq 0$, the kinetic energy $(p+\hbar k)^{2} / 2 M$ of $\left|g_{+}, p+\hbar k\right\rangle$ differs from the kinetic energy $(p-\hbar k)^{2} /$ $2 M$ of $|g-, p-\hbar k\rangle$ by an amount $2 \hbar k p / M$ (i.e., the Doppler shift introduced above for the two-photon Raman resonance).

We can now write the expression of the nonabsorbing trapping state considered above:

$$
\left|\psi_{\mathrm{NA}}(0)\right\rangle=\left[\left|g_{-},-\hbar k\right\rangle+\left|g_{+},+\hbar k\right\rangle\right] / \sqrt{2} \text {. }
$$

This state is stationary since the two states $\left|g_{ \pm}, \pm \hbar k\right\rangle$ have the same internal and kinetic energies, and since $\left\langle\psi_{\mathrm{NA}}(0)|V| e_{0}, 0\right\rangle=0$. These properties are not modified when spontaneous emission is taken into account $\left[\left|\psi_{\mathrm{NA}}(0)\right\rangle\right.$ is radiatively stable], so that an atom pumped in this state remains trapped there indefinitely (coherent population trapping). Note that $\left|\psi_{\mathrm{NA}}(0)\right\rangle$ is not an eigenstate of $P_{z}^{\text {at }}$, so that, for atoms trapped in $\left|\psi_{\mathrm{NA}}(0)\right\rangle$, the atomic momentum distribution presents two peaks at eigenvalues $p_{\text {at }}= \pm \hbar k$.

For the families $F(p \neq 0)$, we can introduce two orthogonal linear combinations of $\left|g_{ \pm}, p \pm \hbar k\right\rangle$ :

$$
\begin{aligned}
& \left|\psi_{\mathrm{NA}}(p)\right\rangle=\left[\left|g_{-, p}-\hbar k\right\rangle+\left|g_{+}, p+\hbar k\right\rangle\right] / \sqrt{2}, \\
& \left|\psi_{\mathrm{A}}(p)\right\rangle=\left[\left|g_{-, p}-\hbar k\right\rangle-\left|g_{+}, p+\hbar k\right\rangle\right] / \sqrt{2} .
\end{aligned}
$$

The first one, $\left|\psi_{\mathrm{NA}}(p)\right\rangle$, is not coupled to $\left|e_{0}, p\right\rangle$, while $\left|\psi_{\mathrm{A}}(p)\right\rangle$ is coupled to $\left|e_{0}, p\right\rangle$ with a Rabi frequency $\sqrt{2} \omega_{1}$. However, the nonabsorbing state $\left|\psi_{\mathrm{NA}}(p)\right\rangle$ is not a trapping state, because it is not stationary (the energies of $\left|g_{ \pm}, p \pm \hbar k\right\rangle$ differ by $\left.2 \hbar k p / M\right)$. More precisely, if an atom is in $\left|\psi_{\mathrm{NA}}(p)\right\rangle$ at $t=0$, it will oscillate between $\left|\psi_{\mathrm{NA}}(p)\right\rangle$ and the absorbing state $\left|\psi_{\mathrm{A}}(p)\right\rangle$ at the frequency $2 \mathrm{kp} / \mathrm{M}$. One can then show that for small values of $p\left[k p / M \ll \Gamma^{\prime}\right.$ where $\Gamma^{\prime} \simeq \omega_{1}^{2} / \Gamma$ is the absorption rate from $\left|\psi_{\mathrm{A}}(p)\right\rangle$ for $\omega_{1} \ll \Gamma$ and zero detuning], the absorption rate from $\left|\psi_{\mathrm{NA}}(p)\right\rangle$ is of the order of $\Gamma^{\prime \prime}=(\mathrm{kp} /$ $M)^{2} / \Gamma^{\prime}$. The smaller $p$, the longer the time spent in $\left|\psi_{\mathrm{NA}}(p)\right\rangle$. We have thus achieved a velocity-selective coherent population trapping. ${ }^{10}$

So far, we have only considered the evolution of a given $p$ family. Spontaneous emission can actually redistribute atoms between different families since the onephoton recoil momentum along $0 z$ due to such a process is a random variable between $-\hbar k$ and $+\hbar k$. Such a random walk in momentum space is essential for the cooling discussed here. It provides the mechanism for the pumping and accumulation of atoms into the nonab- sorbing superposition of states $\left|\psi_{\mathrm{NA}}(p)\right\rangle$ with $p=0$ or very small. The longer the interaction time $\Theta$, the narrower the range $\pm \delta p$ of values of $p$ around $p=0$ for the states $\left|\psi_{\mathrm{NA}}(p)\right\rangle$ in which the atoms can remain trapped during $\Theta$, and the greater the number of fluorescence cycles which can bring them into these states. For $\Theta$ large enough so that $\delta p \leq \hbar k$, the final atomic momentum distribution $\mathcal{P}\left(p_{\text {at }}\right)$ along $0 z$ will exhibit two resolved peaks emerging at $\pm \hbar k$ above the initial distribution. This will be the signature of cooling by velocity-selective coherent trapping.

We have performed a quantitative calculation of such a "generalized optical pumping cycle" (in both internal and momentum spaces) which confirms all the previous predictions. Such a calculation is based on three-level generalized optical Bloch equations involving internal and external degrees of freedom. ${ }^{11}$ Because of spontaneous emission, these equations are finite-difference equations. It must be emphasized that, since the width $\delta p$ can become smaller than $\hbar k$, most of the standard approximation methods used in laser-cooling theories ${ }^{3,8}$ break down: Especially, it is no longer possible to derive a Fokker-Planck equation. Figure 2 shows the final distribution $\mathcal{P}\left(p_{\text {at }}\right)$ of atomic momentum deduced from a numerical integration of Bloch equations for parameters corresponding to our experimental conditions. As expected, one clearly sees two narrow peaks emerging above the background around $\pm \hbar k$. Note that the half-width of each peak is narrower than the one-photon recoil energy. We have checked that an increase of the interaction time $\Theta$ increases the height and decreases the width of these peaks. The value of $\Theta$ leading to the largest area under the peaks depends on the shape of the ini-

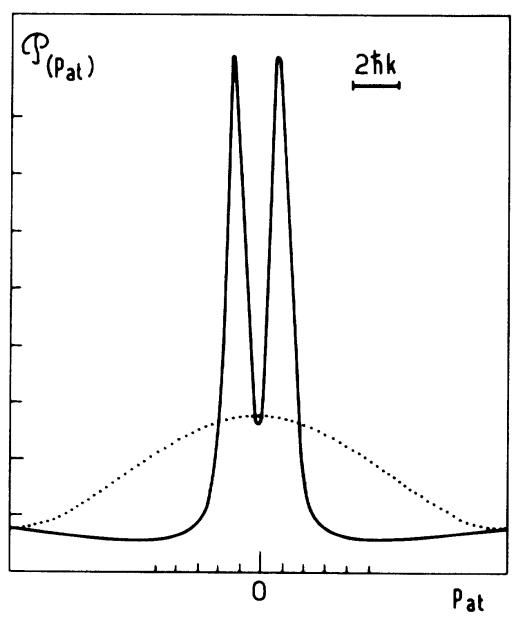

FIG. 2. Calculated transverse atomic momentum distribution resulting from cooling by velocity-selective coherent population trapping, for parameters close to our experimental situation (zero detuning, Rabi frequency $\omega_{1}=0.6 \Gamma$, interaction time $\left.\Theta=350 \Gamma^{-1}\right)$. The initial distribution is represented by a dotted line. 
tial distribution.

This cooling process has been demonstrated with the experimental setup shown in Fig. 3. A supersonic helium beam, ${ }^{12}$ liquid-nitrogen cooled, is excited by counterpropagating electrons at $40 \mathrm{eV}$. The metastable $\mathrm{He}^{*}$ atoms in the $2{ }^{1} S_{0}$ state are optically quenched, and we obtain a beam of $\mathrm{He}^{*}$ in the $2^{3} S_{1}$ state, with an intensity larger than $10^{12}$ atoms $\mathrm{s}^{-1} \mathrm{sr}^{-1}$ and an average velocity of $1100 \mathrm{~m} \mathrm{~s}^{-1}$. The $\mathrm{He}^{*}$ atoms interact on the $2{ }^{3} S_{1}-2^{3} P_{1}$ transition $(1.083 \mu \mathrm{m})$ with a home-made single-mode ring version of a LNA laser ${ }^{13}$ pumped by a $4-\mathrm{W} \mathrm{Ar}^{+}$ laser. The laser frequency is locked to the atomic transition in an auxiliary discharge, by saturated-absorption techniques, and the laser linewidth is less than $1 \mathrm{MHz}$. After spatial filtering, the laser beam is expanded, passed through two quarter-wave plates (Fig. 3), and retroreflected, yielding two counterpropagating plane waves with opposite circular polarizations, with an almost uniform intensity in the $40-\mathrm{mm}$-diam interaction region (Rabi frequency $\omega_{1}=0.6 \Gamma$ with $\Gamma / 2 \pi \simeq 1.6 \mathrm{MHz}$ ). There are stringent requirements for this experiment. First, the Zeeman, sublevels $g+$ and $g$ - must remain degenerate in the whole interaction region. This condition is fulfilled by compensation of the magnetic field to less than $1 \mathrm{mG}$ by Helmholtz coils and a Mumetal shield. Second, the relative phase between both laser beams must remain constant in the whole interaction region. This is achieved by our deriving both waves from the same laser beam and by using very high quality optical components for the second quarter-wave plate and for the retroreflecting mirror (wave-front distortion less than $\lambda / 8)$. Also, the exact overlap of the two beams is adjusted to $10^{-5}$ rad by autocollimation techniques. The transverse velocity distribution after the interaction zone is deduced from a transverse scan of an electron multiplier (sensitive to $\mathrm{He}^{*}$ ) with a $100-\mu \mathrm{m}$ entrance slit, placed downstream at $1.4 \mathrm{~m}$ from a first $100-\mu \mathrm{m}$ slit just after the interaction region. The corresponding HWHM

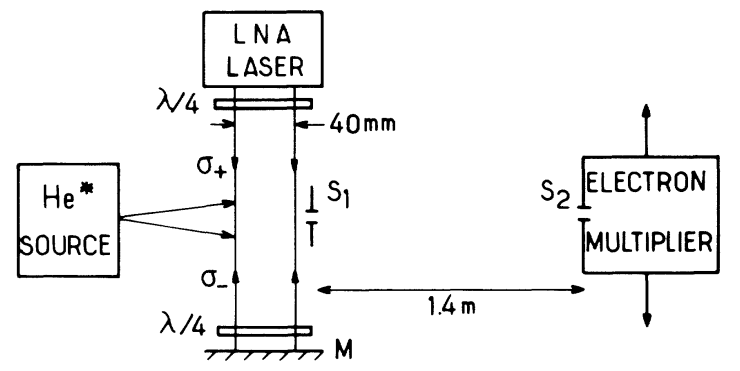

FIG. 3. Schematic experimental setup. The atomic source at $77 \mathrm{~K}$ produces a beam of metastable triple helium atoms $\left(2{ }^{3} S_{1}\right)$ at an average velocity of $1100 \mathrm{~m} / \mathrm{s}$. These atoms interact with two $\sigma_{+}$and $\sigma$ - polarized counterpropagating waves at $1.08 \mu \mathrm{m}$. The transverse velocity distribution at the end of the interaction region is analyzed with two slits $S_{1}$ and $S_{2}, 100$ $\mu \mathrm{m}$ wide. $\mathrm{S}_{2}$ is the entrance slit of a movable $\mathrm{He}^{*}$ detector. transverse velocity resolution is $4 \mathrm{~cm} \mathrm{~s}^{-1}$.

Figure 4 shows the transverse velocity profiles with and without laser. The two peaks at about $\pm \hbar k / M$ $\left( \pm 9.2 \mathrm{~cm} \mathrm{~s}^{-1}\right)$ clearly appear well above the initial distribution. A measurement of the standard half-width at $\exp \left(-\frac{1}{2}\right)$ gives $6 \mathrm{~cm} / \mathrm{s}$, which corresponds to a temperature of about $2 \mu \mathrm{K}$. This experimental curve is in reasonable agreement with the theoretical prediction. Finer details concerning, for example, the variations of the efficiency of the cooling effect with the detuning still require further investigation.

We have performed supplementary tests to support the theoretical analysis given above. First, we replaced the $\sigma+$ and $\sigma$ - circularly polarized beams by two orthogonally linearly polarized beams, and we checked that the final velocity distribution still presents two peaks at $\pm \hbar k / M$. On the contrary, for parallel linear polarizations where the nonabsorbing atomic superposition is not velocity selective, the two peaks at $\pm \hbar k / M$ disappear. Another test consists of our arranging the laser beams so that they do not exactly overlap at the end of the interaction region, the last acting laser beam being the $\sigma+$ one. One expects atoms to be removed from the $\left|g_{-},-\hbar k\right\rangle$ component of $\left|\psi_{\mathrm{NA}}(0)\right\rangle$ and to be pumped after a few cycles (two on the average) into $g_{+}$with a momentum spread around $+\hbar k$. Indeed, we have observed that the peak at $-\hbar k$ disappears while the peak at $+\hbar k$ increases and is broadened.

We have thus demonstrated that this velocity-selective optical pumping into a nonabsorbing state is a very efficient process to accumulate atoms in an extremely narrow velocity class. By increasing the coherent interaction time, still narrower velocity distributions could

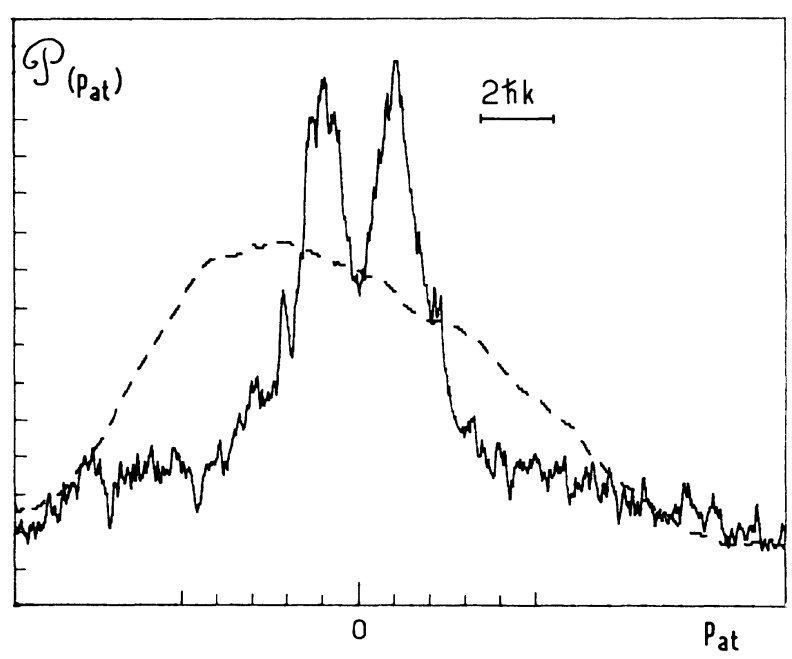

FIG. 4. Transverse atomic momentum profile at the end of the interaction region, with the laser on (solid line) and off (dashed line; this profile has been smoothed). The double-peak structure at about $\pm \hbar k$ and above the initial distribution is a clear signature of the cooling effect presented in this Letter. 
be produced, allowing one to reach temperatures in the nanokelvin range. Several developments of this work can be considered: extensions to other level schemes; direct observation of the coherence between the two components of $\left|\psi_{\mathrm{NA}}(0)\right\rangle$ propagating along different directions; generalization to three dimensions.

Finally, let us emphasize that this cooling mechanism is quite different from the previously demonstrated ones, since it is not due to a friction force but to diffusion into the cooled velocity class. Another important feature is that the cooled atoms no longer interact with the laser field which then causes no perturbation, either on external degrees of freedom (no diffusion) or on internal degrees of freedom (no light shifts). This particularity may be essential for future applications.

We warmly thank our colleagues Jean Dalibard and Christophe Salomon for very useful contributions to this work. This work is part of an operation supported by the stimulation program of European Economic Community. We have benefitted from crucial help from Helmut Haberland and Martin Karrais with the $\mathrm{He}^{*}$ atomic beam. Laboratoire de Spectroscopie Hertzienne de l'Ecole Normale Supérieure is Laboratoire No. 18 associé au Centre National de la Recherche Scientifique.

\footnotetext{
(a) Permanent address: Dipartimento di Fisica, Università di Pisa, I-56100 Pisa, Italy.

${ }^{1}$ D. J. Wineland and W. M. Itano, Phys. Rev. A 20, 1521 (1979); J. P. Gordon and A. Ashkin, Phys. Rev. A 21, 1606 (1980).

${ }^{2}$ H. Dehmelt, G. Janik, and W. Nagourney, Bull. Am. Phys. Soc. 30, 612 (1985); P. E. Toschek, Ann. Phys. (Paris) 10, 761 (1985).

${ }^{3}$ M. Lindberg and J. Javanainen, J. Opt. Soc. Am. B 3, 1008 (1986).

${ }^{4}$ P. D. Lett, R. N. Watts, C. I. Westbrook, W. D. Phillips, P. L. Gould, and H. J. Metcalf, Phys. Rev. Lett. 61, 169
}

(1988).

${ }^{5}$ See the contributions of J. Dalibard et al. and S. Chu, in Proceedings of the Eleventh International Conference on Atomic Physics, edited by S. Haroche, J. C. Gay, and G. Grynberg (World Scientific, Singapore, to be published).

${ }^{6}$ D. E. Pritchard, K. Helmerson, V. S. Bagnato, G. P. Lafyatis, and A. G. Martin, in Laser Spectroscopy VIII, edited by S. Svanberg and W. Persson (Springer-Verlag, Heidelberg, 1987), p. 68

${ }^{7}$ First experimental observation in G. Alzetta, A. Gozzini, L. Moi, and G. Orriols, Nuovo Cimento 36B, 5 (1976); analyses in E. Arimondo and G. Orriols, Lett. Nuovo Cimento 17, 333 (1976); H. R. Gray, R. W. Whitley, and C. R. Stroud, Opt. Lett. 3, 218 (1978).

${ }^{8}$ V. G. Minogin and Yu. V. Rozhdestvenskii, Zh. Eksp. Teor. Fiz. 88, 1950 (1985) [Sov. Phys. JETP 61, 1156 (1985)]. The theoretical treatment of these authors is valid only for atomic momenta $p$ larger than the photon momentum $\hbar k$ since their Fokker-Planck equation is based on an expansion in powers of $\hbar k / p$.

${ }^{9}$ Although we use here a classical description of the laser field, the matrix elements of $\exp ( \pm i k z)$ appearing in $V$ introduce the conservation laws for the total momentum.

${ }^{10}$ Semiclassical arguments can help one to understand the properties of $\left|\psi_{\mathrm{NA}}(p)\right\rangle$. In the laser configuration of Fig. 1(a), the polarization of the total laser electric field $\mathbf{E}_{L}$ is linear and forms a helix along $0 z$ with a pitch $\lambda$. For an atom in $\left|\psi_{\mathrm{NA}}(p)\right\rangle$ the transition dipole moment $\mathbf{d}$ between $\left|\psi_{\mathrm{NA}}(p)\right\rangle$ and $\left|e_{0}, p\right\rangle$ is also linearly polarized and forms a similar helix with the same pitch. The important point is that $\mathbf{d}$ is perpendicular to $\mathbf{E}_{L}$ for all $z$, so that the coupling is zero. Furthermore, the "laser helix" is at rest (since both lasers have the same frequency) whereas the "atomic helix" moves along $0 z$ with a velocity $p / M$. Only for $p=0$ do both helices keep orthogonal polarizations for all times.

${ }^{11}$ Generalized optical Bloch equations have been discussed in detail by R. J. Cook, Phys. Rev. A 22, 1078-1098 (1980).

${ }^{12}$ H. Conrad, G. Ertl, J. Küppers, W. Sesselmann, and H. Haberland, Surf. Sci. 121, 161 (1982).

${ }^{13}$ L. D. Schearer, M. Leduc, D. Vivien, A. M. Lejus, and J. Thery, IEEE J. Quantum Electron. 22, 713 (1986). 